\title{
Anesthetic Management in Neonatal Pediatric Patients with Myelomeningocele and Macrocephaly
}

\author{
Guillermo Vera Duarte ${ }^{1^{*}}$, Paulo Fernando Azuaga Gaona ${ }^{1}$, HéctorFabián Meza Vera $^{2}$ and \\ Edgar Walter LópezSamaniego ${ }^{3}$ \\ ${ }^{1}$ Resident of Anesthesiology and Resuscitation at the Hospital de Clínicas, San Lorenzo, Paraguay. \\ ${ }^{2}$ Director of Post-degree of Anesthesiology of the Faculty of Medical Sciences, Anesthesiologist staff at National University of Asunción, Paraguay.
}

${ }^{3}$ Anesthesiologist staff at National University of Asunción, Paraguay.

\begin{abstract}
Received: January 19 2018; Accepted: July 19 2018; Published: November 142018
*Corresponding author: Guillermo Vera Duarte, Resident of Anesthesiology and Resuscitation at the Hospital de Clínicas, San Lorenzo, Paraguay, E-mail: guillermoveraduarte@gmail.com
\end{abstract}

\section{Abstract}

Introduction: Anesthetic therapies in neonatal patients with Myelomeningocele and Macrocephaly have important points to keep in mind. Myelomeningocele (MMC) represents the most severe form of spinal dysraphism. Early surgical treatment was associated with a lower rate of complications.

Report: Neonatal term patient, suitable for gestational age, with diagnoses of lumbo-sacral myelomeningocele, bilateral bot foot, Macrocephaly and pyelocalic dilatations in both kidneys.

Conclusions: Anesthesia of the patient with multiple malformations poses an anesthetic challenge. The neurological deficit can be minimized through careful attention to the preoperative evaluation, good surgical technique, good hemodynamic control and the subsequent control of a multidisciplinary team to be attentive to complications that may present.

Keywords: Myelomeningocele; Neonatal; Anesthesia; Monitoring;

\section{Introduction}

It is a congenital anomaly of the neural tube in which the bones of the spine do not form completely and the canal is incomplete, which allows the spinal cord and menins to protrude through the back of the child, the marrow forms a plaque and the meninges they are reduced to the pia mater, in front of the marrow. The CSF pushes through the bone dehiscence. This nervous tissue is in contact with an intermediate zone, a thin membrane, called the transitional epithelium; when this membrane does not exist, the nervous tissue is in contact with the skin. (favoring the risk of infection and damage to the central nervous system). This pathology usually includes 3 to 6 vertebral segments [1].

Paraguay registers a national registry of congenital malformations to know the prevalence of this pathology. However, the isolated studies of public hospitals reveal that they resemble the average of what is known by the literature that speaks a reality between 0.2 and 2.0 per 1,000 live births, with regional and racial variations [2].
These are some of the cases in which the brain and Chiari syndrome type II, which involves the herniation of structures from the lower part of the cerebellum and brain stem through the human body, that some parts of the brain reach the spinal canal thickening and compressing it [3].

Early surgical treatment ( $\leq 5$ days) was associated with a shorter length of hospital stay and antibiotic therapy, as well as a lower rate of complications [4]. It reduces neurological damage and complications as an infection, as well as to help the family cope with this disorder.

Before surgery, the child must be treated with care to reduce the damage to the exposed spinal cord, which includes a special position, protective devices and modifications in the way of feeding, handling, bathing and caring for the baby. The tensionfree closure of the skin in the midline is still the preferred technique, for which in most cases it is necessary to dissect in depth the edges of the skin surrounding the defect. However, large defects cannot be reliably closed with this technique and it is essential that in case of using a flap this has a good blood supply, with a sufficient thickness to protect the underlying neuronal structure, and a repair to prevent leakage of cerebrospinal fluid [5]. In cases of associated hydrocephalus, some authors performed a ventriculoperitoneal shunt in the same act, early surgical treatment ( $\leq 5$ days) was associated with a shorter length of hospital stay and antibiotic therapy, as well as a lower rate of complications.

\section{Case Report}

Newborn patient of term, suitable for gestational age, of 12 hours of life, male with diagnosis of lumbo-sacral myelomeningocele, bilateral bot foot, macrocephaly and pyelocalic dilatations in both kidneys in myelomeningocele closure plans and placement of a ventriculoperitoneal shunt catheter. 
Arrives in a servo-assisted operating room, with nasogastric tube at the slope, peripheral venous line in the right arm with punzocath No. 24 with $10 \%$ Dextrose drip at $9 \mathrm{cc} / \mathrm{h}$ per pump and left arm with punzocath No. 24 sealed, micro dripper connected with physiological solution. It is placed on the operating table on a thermal blanket, monitoring includes pulse oximetry with Massimo ${ }^{\circledR}$ monitor, non-invasive tensiometer, 5 -lead cardioscopy.
Anesthetic induction, with intravenous administration fentanyl 10 micrograms, atracurium $1 \mathrm{mg}$ administered with 1 $\mathrm{ml}$ of jerga and sevoflurane $3 \%$, orotracheal intubation with tube No. 3.5 without cuff, is added to the esophageal temperature monitoring, a neonatal bladder catheter is installed. The patient is placed in the ventral decubitus corroborating the proper position of the orotracheal tube and PA at each patient's movement. Maintenance of temperature by thermal blanket and heating of intravenous fluids (Figure 1).

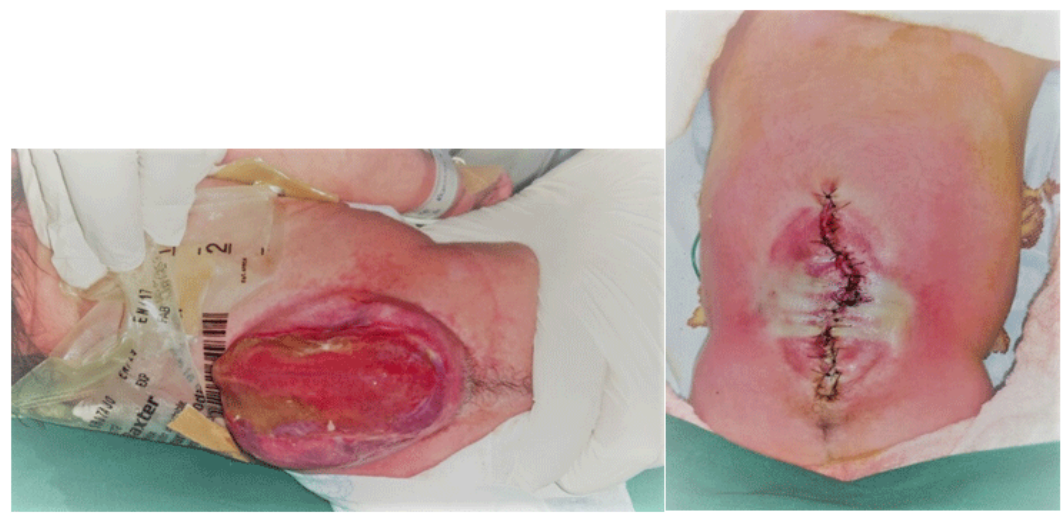

Figure 1:

Left: Defect of the pre-surgical column.

Right: Postoperative defect closure.

Maintenance of anesthesia with sevoflurane 2\%, infusion of remifentanil at $0.1 \mathrm{mcg} / \mathrm{kg} / \mathrm{min}$, atracurium $0.5 \mathrm{mg} / \mathrm{kg} / \mathrm{h}$.

Patient with stable hemodynamics throughout the procedure, physiological solution was administered at $40 \mathrm{cc} / \mathrm{kp}$, neutral thermal environment, diuresis $1 \mathrm{cc} / \mathrm{kp} / \mathrm{h}$, did not require vasoactive drugs.

Analgesia by local infiltration with bupivacaine $0.25 \%$ at $1 \mathrm{mg} . \mathrm{kg}$ of the puncture sites for the placement of the bypass catheter and paracetamol IV at 15 mg.kg every 6 hours. The duration of the surgery was 3 hours and a half, the patient is extubated without complications after 4 hours from intubation and transferred to the NICU.

\section{Discussion}

Meningoceles and myeloceles are usually repaired within the first day of life to minimize bacterial contamination of the exposed spinal cord and subsequent sepsis, which is the most common cause of death in this population during the period of the newborn.

Anesthesia of the patient with multiple malformations poses an anesthetic challenge, both due to the hemodynamic implications due to the loss of volume, as well as changes in position that increase the frequency of events (loss of tracts, endotracheal tube malposition or accidental extubation, loss of temperature). There are several aspects that should be considered in neonatal anesthesia and that our case shows them:
Invasive monitoring Invasive monitoring in the newborn is a challenge and with a high frequency of complications. One of the recommended options is to use the NIRS as a non-invasive measure whose value is modified by changes in tissue perfusion, helps diagnosis and serves to guide decision-making, which we did not have.

Position of the patient, three changes of surgical position are necessary during the intervention. The mobilizations must be careful. In our case we performed an intubation in dorsal decubitus with cervical control. The occipital area was protected by an impeller, trying to minimize the effect on intracranial pressure. In prone it is placed on the face with two cylinders padded in the roots of the limbs and cotton in prone areas. Repeated changes in the surgical position are accompanied by malposition of the endotracheal tube, potential intubation and loss of patient temperature.

Analgesia, alterations in the sensitivity of myelomeningocele should be considered but the use of analgesic drugs should not be different. It can also acquire memory in front of said stimulus through hyperalgesia mechanisms and trigger immediate defensive responses to it. For all this, you should treat the pain associated with the surgery as soon as possible and, if possible, prevent it before it even occurs. Paracetamol IV can be administered in neonatal patients younger than 1 month of age at a dose of $15 \mathrm{mg} . \mathrm{kg}$ every 6 hours. Also take into account the safe dose of Bupivacaine at $1 \mathrm{mg} . \mathrm{kg}$. 
Control of the temperature, the surgical exposure of different cavities, as well as the repeated changes of position, entail a great loss of heat. In our case, thermal blanket and esophageal temperature control were used.

\section{Conclusion}

The neurological deficit can be minimized by careful attention to the preoperative evaluation, good surgical technique, early intervention ( $\leq 5$ days) accompanied by a good hemodynamic control using cardiovascular monitoring, temperature monitoring and continuous monitoring of spinal cord function.

\section{References}

1. Goldschmidt EL and Tello AM. Prevention of neural tube closure defects. Journal of the Children's Hospital of Buenos Aires. 2000;42:238-244.

2. Mansouri HA. Central nervous system anomalies diagnosed antenatally and post-delivery management. Saudi Med J. 2010; 31(3):257-61.

3. Canún-Serrano S, Zafra-De la Rosa G. Detection of external congenital malformations. Bol Med Hosp Inf Mex 1984;41:21.

4. Oncel Mehmet Yekta, Ramazan Ozdemir, Gokmen Kahilogulları, Sadık Yurttutan, Omer Erdeve and Ugur Dilmen. "The Effect of Surgery Time on Prognosis in Newborns with Meningomyelocele." Journal of Korean Neurosurgical Society. 2012;51(6):359-362. Doi: 10.3340 / jkns.2012.51.6.359.

5. Nejat Farideh, Nima Baradaran and Mostafa Ei Khashab. Large myelomeningocele repair. Indian Journal of Plastic Surgery. 2011;44(1):87-90. Doi: 10.4103 / 0970-0358.81453. 